\title{
Une méthode simple pour le calcul des ondes de rupture de barrage
}

\author{
André Paquier, Olivier Robin \\ CEMAGREF *
}

\section{I $\square$ INTRODUCTION}

La rupture d'un barrage est un événement rare mais d'ampleur parfois considérable. Chaque année, dans le monde, en moyenne, 1 à 2 barrages de plus de 10 mètres de haut ont des problèmes tels qu'ils occasionnent la rupture partielle ou totale de l'ouvrage. En France, grâce, en particulier, aux mesures prises suite à la catastrophe de Malpasset, ces 30 dernières années n'ont vu aucune rupture d'importance notoire. Il demeure que, avant même qu'un risque réel de rupture se manifeste, il est nécessaire de connaître les zones éventuellement submergées suite à une rupture afin de, par exemple, préparer un plan d'évacuation des populations concernées.

Pour les grands barrages (plus de 20 mètres de haut et 15 millions de $\mathrm{m}^{3}$ ), la réglementation française prévoit la procédure de plan d'alerte. Pour les ouvrages de moindre importance, aucune disposition n'est obligatoire mais il importe de déterminer les zones susceptibles d'être submergées (et les caractéristiques de l'ondé dans ces zones) afin de pouvoir juger si la sécurité publique est en cause et éventuellement prévoir des dispositions particulières.

Pour la détermination des zones submergées, les moyens de calcul modernes ont permis d'apporter des réponses diversifiées. Les méthodes de calcul sont, en général, constituées de 2 étapes séparées :

- la simulation du phénomène de rupture de l'ouvrage qui permet d'obtenir l'hydrogramme au droit du barrage ; - la simulation de la propagation de l'onde dans la vallée à l'aval qui donnera, en général, en tout point, les carac-

(*) Division Hydrologie-Hydraulique, 3 bis, quai Chauveau CP 220, 69336 Lyon Cedex 09. téristiques suivantes : la hauteur d'eau maximale, la vitesse maximale et le temps d'arrivée de l'onde.

En ce qui concerne la première étape de rupture de l'ouvrage ou plus exactement l'obtention de l'hydrogramme au droit du barrage, les méthodes sont très diverses. On peut distinguer :

a) celles qui supposent une rupture instantanée (totale ou partielle) de l'ouvrage avec soit utilisation d'une formule simplifiée, soit calcul de ligne d'eau ;

b) celles qui supposent une rupture progressive de l'ouvrage avec soit variation linéaire des caractéristiques de la brèche, soit utilisation d'un modèle d'érosion ;

c) celles qui partent d'une analyse statistique des ruptures réellement observées pour obtenir une relation entre le débit de pointe au droit du barrage et différentes caractéristiques du barrage ou de la retenue (en général, hauteur du barrage et volume de la retenue).

Pour les digues en terre, les 3 méthodes sont utilisées, un calcul de type b étant le plus précis et le plus complexe. Pour les ouvrages en béton, seules les méthodes de type a sont à considérer, les ruptures observées ayant été très rapides (durée de l'ordre de la minute).

En ce qui concerne la seconde étape de propagation, la même simplification du phénomène réel est admise par la plupart ; on se contente de modéliser le seul écoulement de l'eau dans l'axe de la vallée sans tenir compte de la complexité du processus physique qui inclut des accélérations transversales et verticales souvent importantes (au niveau du front) et un transport solide intense. La modélisation s'effectue par un des moyens suivants :

a) résolution des équations de Saint-Venant ;

b) utilisation d'abaques (ou relations entre grandeurs adimensionnelles) basés sur une généralisation de résultats des méthodes de type a);

\section{A simple method for the computation of dam-break waves}

We describe a simplified method for computing dam-break waves and the results of the corresponding validation tests. Computation for one given cross section only uses data relative to the dam and that section completed by the Manning-Strickler coefficient for the reach between the dam and that section. The references of the various comparisons consist of computations with a model solving de Saint-Venant equations. Results are generally good as well for maximum water heights (difference less than $30 \%$ in more than $90 \%$ of sections) as for maximum velocities and wave arrival times. We also note this important point that the uniform flow equation is verified between maximum discharge and maximum water height. 


\begin{tabular}{|l|l|}
\hline \multicolumn{2}{|c|}{ Notations } \\
\hline I pente de la vallée à l'abscisse $X$ & $V_{0}$ volume de la retenue \\
$K$ coefficient de Strickler & $V_{\text {MAX vitesse maximale à l'abscisse } X}$ \\
$Q B_{\text {MAX débit de pointe au droit du barrage }}$ & X distance d'un point jusqu'au barrage \\
$Q_{\text {MAX }}$ débit maximal à l'abscisse $X$ & $Y_{\text {MAX }}$ hauteur d'eau maximale à l'abscisse $X$ \\
\hline
\end{tabular}

c) résolution d'équations obtenues à partir des équations de Saint-Venant par simplification de l'équation dynamique.

De ces 3 méthodes, la plus précise est la première mais elle est aussi d'une complexité de mise en œuvre bien supérieure.

\section{II 口 OBJECTIFS DE LA MÉTHODE MISE AU POINT}

Au cours des 10 dernières années, le CEMAGREF a développé plusieurs logiciels de calcul pour répondre à la demande française en matière de calcul d'onde de rupture de barrage. L'objectif était clairement de disposer des outils les plus performants. Ont donc été conçus un logiciel (RUPRO) de simulation d'érosion de digue en terre (par surverse ou par renard) ainsi qu'un logiciel (RUBAR 3) de résolution des équations de Saint-Venant en présence de front. Récemment, un logiciel (RUBAR 20) a vu le jour pour la résolution des équations de Saint-Venant bidimensionnelles.

Ces outils relativement complexes ne peuvent être mis en œuvre que par des bureaux d'études spécialisés ; leur emploi ne peut être généralisé à l'ensemble des services qui ont à gérer les problèmes de sécurité des barrages. Sauf circonstances particulières, les méthodes décrites ci-dessus sont donc réservées aux grands barrages, une méthode simplifiée devant être utilisée pour les ouvrages de moindre importance.

Cette méthode devrait répondre aux critères suivants :

- être d'une mise en œuvre suffisamment simple pour qu'un ingénieur n'ayant que des connaissances de base en hydraulique puisse l'utiliser rapidement et sans risque d'erreur excessif ;

- s'appliquer à la majeure partie des cas ;

- présenter des résultats avec une marge d'incertitude la plus réduite possible. Dans le cas des grands barrages, le Comité Technique Permanent des Barrages (C.T.P.B.) admet une incertitude de l'ordre de $15 \%$ sur les résultats en hauteurs d'eau et temps. Avec une précision identique sur les données, l'incertitude sur les résultats d'une méthode simplifiée devrait se situer entre 20 et $50 \%$.

Un tour d'horizon des méthodes existantes nous a conduit au constat suivant :

- le CTGREF (Centre Technique du Génie Rural des Eaux et des Forêts) avait présenté en 1978 [1] une méthode par abaques encore largement utilisée mais qui avait pour principal inconvénient de ne s'appliquer qu'aux ruptures instantanées ;

- des différentes méthodes américaines, le modèle SMPDBK du NWS (National Weather Service) apparaissait comme le plus précis et le plus facile d'emploi [6]. Des tests sur des barrages français [4], ont toutefois montré que l'erreur sur les résultats pouvait dépasser $100 \%$ (par comparaison à des calculs menés avec un calcul de type Saint-Venant) et était souvent supérieure à celle de la méthode par abaques du CTGREF.

Nous avons donc décidé de reprendre cette dernière méthode afin de l'améliorer sur les points suivants :

- extension aux ruptures progressives ;

- extension du domaine de validité ;

- augmentation de la précision sur les résultats :

- intégration dans un logiciel d'utilisation simple.

\section{III — VALIDATION DU LOGICIEL CASTOR}

Le logiciel ainsi créé et dénommé CASTOR (CAlcul Simplifié pour le Traitement des Ondes de Rupture de barrage) repose sur les 5 étapes de calcul suivantes :

1) calcul du débit de pointe au droit du barrage $Q B_{\operatorname{MAX}}$ par des formules simples différentes en rupture progressive et en rupture instantanée ;

2) détermination, grâce à un abaque numérisé, du rapport $Q_{\mathrm{MAX}} / Q B_{\mathrm{MAX}}$ du débit maximal en une section quelconque à ce débit de pointe au barrage ;

3) calcul de la hauteur d'eau maximale $Y_{\operatorname{MAX}}$ à partir de $Q_{\mathrm{MAX}}$ par l'équation du régime uniforme ;

4) calcul de la vitesse maximale $V_{\operatorname{MAX}}$ à partir de $Q_{\mathrm{MAX}}$ et de la section mouillée correspondant à $Y_{\mathrm{MAX}}$;

5) calcul du temps d'arrivée de l'onde à partir de $V_{\text {MAX }}$ et de la distance au barrage.

Nous examinons ci-dessous les résultats de tests de validation effectués successivement pour chacune des principales étapes. Ces tests ont consisté à comparer les résultats de CASTOR à ceux de RUBAR 3 qui résout les équations de Saint-Venant. Ceci a été effectué sur un échantillon de 15 barrages totalisant 440 sections en travers sur environ 500 kilomètres de vallée à l'aval des barrages. Cet échantillon constitué d'ouvrages pour lesquels le problème de sécurité a été réellement posé recouvre un éventail très large de situations quant à la topographie de la vallée (pente de 0,01 à $10 \%$ ) et à l'importance de l'ouvrage (volume de retenue de $730000 \mathrm{~m}^{3}$ à 48 millions de $\mathrm{m}^{3}$, hauteur du barrage de 8 à 60 mètres).

\subsection{Débit de pointe au droit du barrage}

En rupture instantanée, $Q B_{\mathrm{MAX}}$ est déterminé par la formule de Ritter corrigée par un coefficient qui tient compte de la forme de la retenue (plus ou moins allongée). Le coefficient utilisé par la méthode par abaques [1] a été 
légèrement modifié car il conduisait à sous-estimer $Q B_{\mathrm{MAX}}$. Le résultat de l'ajustement est présenté à la figure $I$ sur les 7 barrages de l'échantillon traités en rupture instantanée.

En rupture progressive, CASTOR utilise une formule simplifiée d'origine statistique calée sur des observations de rupture [3]. Aucune comparaison n'est ici effectuée entre les résultats donnés par cette formule et d'éventuels autres résultats, aucune référence indiscutable n'existant en ce domaine. Les comparaisons globales présentées ci-après supposeront donc en rupture progressive même débit maximal au barrage dans CASTOR que dans RUBAR 3 .

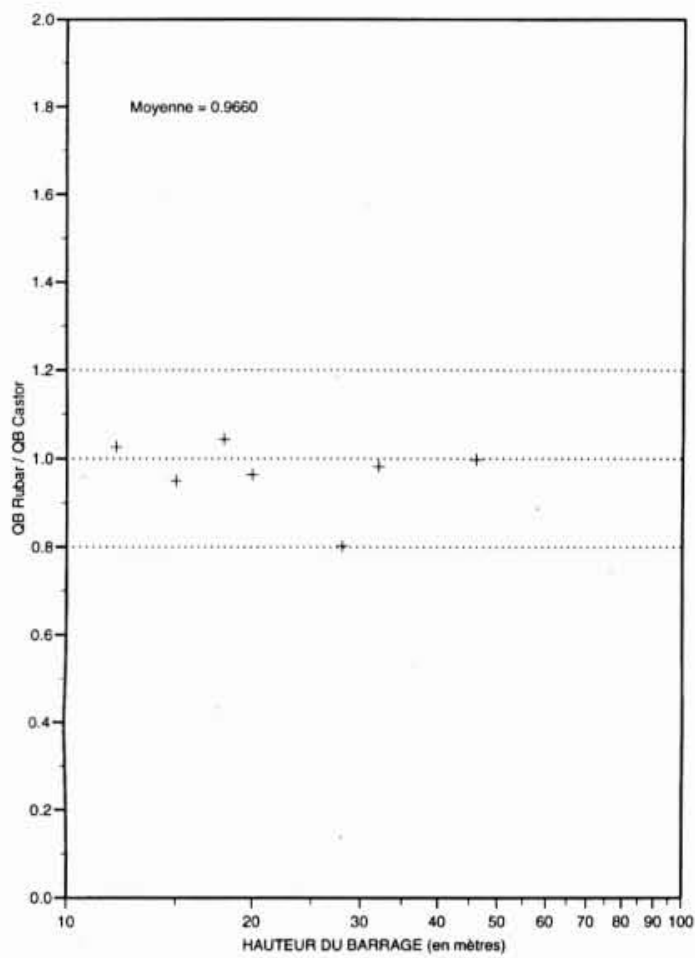

1. Débits de pointe au barrage en rupture instantanée.

\subsection{Débit maximal dans une section}

On utilise un abaque constitué d'un faisceau de courbes correspondant aux différentes valeurs du produit $I K^{2}$ de la pente par le carré de Strickler (données moyennes entre le barrage et la section en question). Connaissant la valeur de $I K^{2}$, l'abaque permet de déterminer le rapport $Q_{\mathrm{MAX}} / Q B_{\mathrm{MAX}}$ en fonction de la variable adimensionnelle $X / V_{0}^{1 / 3}, V_{0}$ représentant le volume de la retenue en $\mathrm{m}^{3}$ et $X$ la distance au barrage en mètres. Afin de pouvoir traiter le maximum de cas, nous sommes partis de l'abaque du CTGREF [1] et avons étendu son domaine de validité en effectuant des simulations à partir de RUBAR 3 sur un canal triangulaire de pente et de Strickler constants.

Ces calculs ont permis de compléter l'éventail de courbes $I K^{2}$ disponibles entre 0,1 et 64, l'abscisse maximale (en variable adimensionnelle $X / V_{0}^{1 / 3}$ ) étant portée à 700 , ce qui donne, par exemple, $70 \mathrm{~km}$ pour une retenue de 1 million de $\mathrm{m}^{3}$.
Cet abaque reste valable uniquement pour des ruptures instantanées. Le cas des ruptures progressives est traité en supposant qu'un hydrogramme de rupture progressive est analogue à un hydrogramme de rupture instantanée déjà amorti. On définit ainsi un bief fictif à l'amont du barrage réel de pente et de coefficient de Strickler représentatifs de la vallée réelle et de longueur suffisante pour que si le barrage se rompait instantanément à l'amont de ce bief fictif, le débit maximal résultant au droit du barrage réel correspondrait au débit de pointe de la rupture progressive.

La figure 2 montre à même débit de pointe au barrage les résultats en terme de débit maximal en chaque section en travers.

La dispersion est limitée (écart type : 0,24) avec une moyenne proche de 1 que ce soit la «moyenne par section » (qui correspond à une moyenne effectuée avec le même poids pour chaque section en travers) ou la " moyenne par barrage " (qui correspond au même poids donné à la moyenne de chaque barrage, chaque section d'un même barrage étant crédité du même poids).

Nous avons aussi examiné séparément les résultats en rupture instantanée et progressive. On remarque alors une légère surestimation de $Q_{\mathrm{MAX}}$ par CASTOR en rupture instantanée. Les résultats y sont, par ailleurs, plus dispersés qu'en rupture progressive.

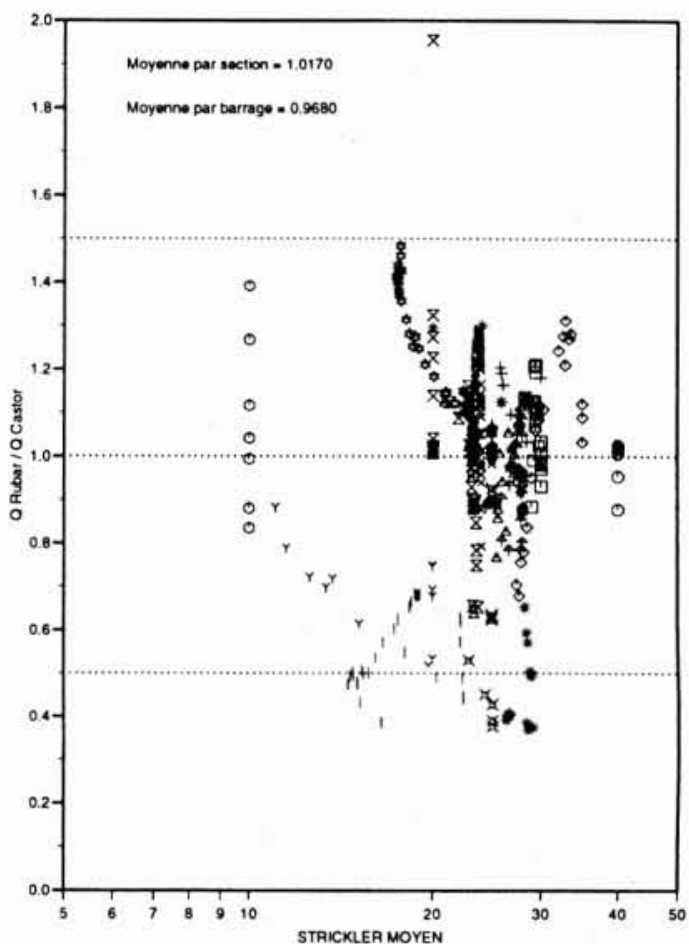

2. Vérification de l'abaque donnant le débit maximal.

\subsection{Hauteur d'eau maximale}

Le calcul de la hauteur d'eau maximale est effectué à partir de la description en couples largeur-cote de la section en travers, d'un Strickler local et d'une pente locale donnés par l'utilisateur. On suppose le régime uniforme (pente égalée au frottement) afin d'obtenir une relation locale (indépendante des autres sections) entre le débit et la hauteur d'eau. 
Dans le cas de faibles pentes ou contrepentes, on considère une pente locale minimale de $0,05 \%$. Dans le cas de singularités topographiques (rétrécissements,...), la hauteur d'eau maximale étant influencée par la topographie voisine, on permet d'effectuer une description plus fine de la topographie en ajoutant 1 ou 2 sections en travers à la description du point où s'effectue le calcul ; ces sections supplémentaires permettent de définir une section en travers moyenne plus représentative de la situation locale. La figure $3 \mathrm{~A}$ montre que, moyennant ces précisions (appliquées sur notre échantillon, à environ 30 sections), à $Q_{\mathrm{MAX}}$ donné, l'équation du régime uniforme (le frottement exprimé en fonction de $Y_{\mathrm{MAX}}$ et $Q_{\mathrm{MAX}}$ est égalé à la pente) permet de retrouver une bonne approximation de $Y_{\mathrm{MAX}}$; la moyenne est proche de 1 et l'écart type de l'ordre de 0,11 .

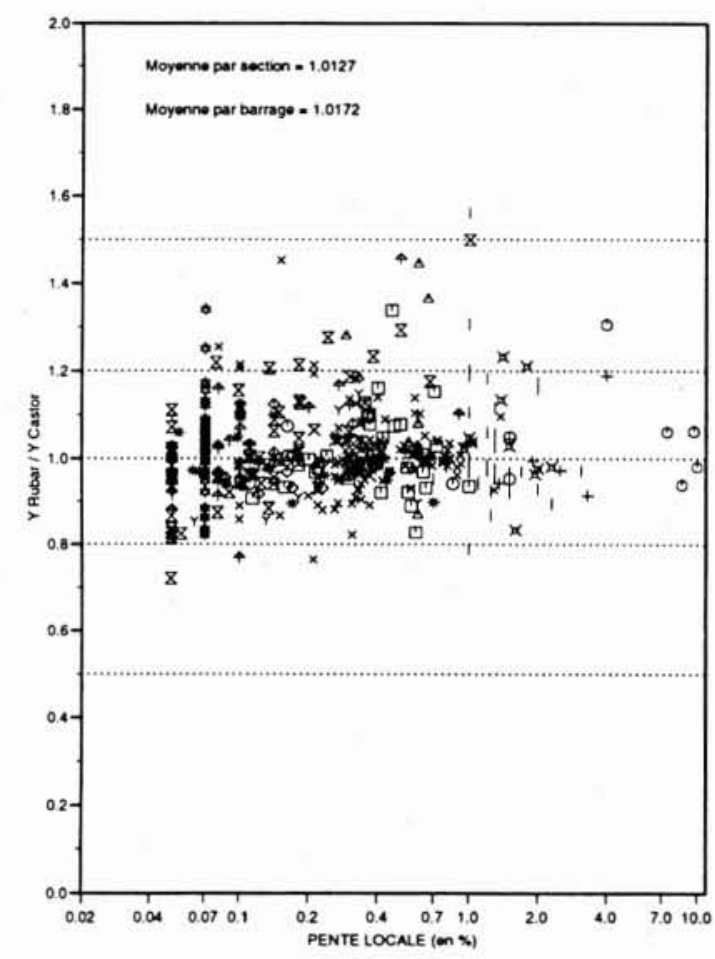

3A. Vérification de l'équation du régime uniforme.

A noter que les hauteurs d'eau sont en moyenne légèrement surestimées par CASTOR dans le cas des sections mises à la pente minimale de $0,05 \%$ et sous-estimées dans le cas des 11 sections où des sections supplémentaires ont été utilisées. Sans emploi de ces sections supplémentaires, la moyenne augmente de 0,004 et l'écart-type passe à 0,13 ; on trouve encore $93 \%$ de résultats donnant un rapport compris entre 0,8 et 1,2 .

$\mathrm{Si}$ on compare les résultats globaux sur les 3 premières étapes du calcul, on obtient également (cf. figure $3 B$ ) un nuage très dense (écart-type: 0,15 ) avec une moyenne proche de 1 . On observe donc 2 phénomènes : d'une part, une certaine compensation des erreurs commises dans chacune des étapes de calcul successives et, d'autre part, une diminution du pourcentage d'erreur sur $Y_{\mathrm{MAX}}$ par rapport au pourcentage d'erreur sur $Q_{\mathrm{MAX}}$.

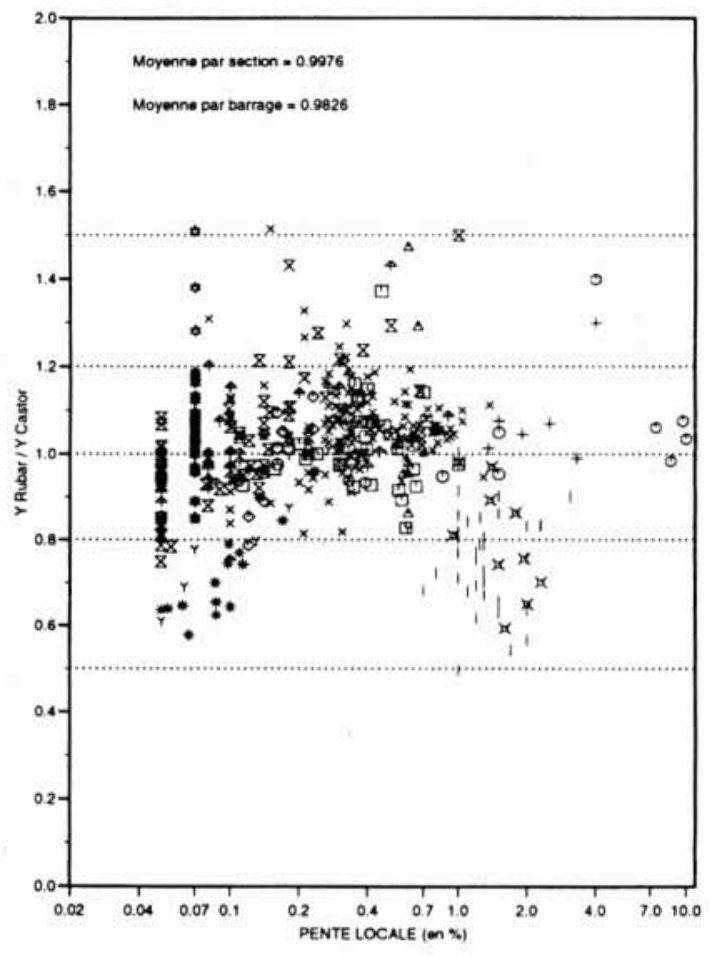

3B. Hauteurs d'eau maximales.

\subsection{Vitesses maximales}

Pour obtenir la vitesse maximale moyenne en une section, on considère la vitesse obtenue par le calcul précédent en régime uniforme à laquelle on applique un coefficient correcteur de 1,2 qui permet de tenir compte du fait que la vitesse à l'arrivée du front est, en général, plus forte que plus tard alors que niveau d'eau ou débit sont maximaux.

La référence du logiciel RUBAR 3 est toujours utilisée mais elle est ici plus critiquable car des vitesses maximales différentes peuvent être obtenues selon la hauteur d'eau minimale qui est considérée pour prendre en compte cette vitesse.

On observe que si, grâce au coefficient correcteur, la moyenne est toujours proche de 1, la dispersion est beaucoup plus grande (écart-type : 0,34 ), une dizaine de points (non mentionnés sur la figure 4) donnant même des rapports supérieurs à 2 . Ce calcul donne toutefois un bon ordre de grandeur $(90 \%$ des résultats entre 0,5 et 1,5$)$ de la vitesse maximale et donc du danger potentiel lié aux fortes vitesses.

\subsection{Temps d'arrivée de l'onde}

Comme pour la vitesse maximale, il est ici difficile de trouver une bonne référence pour cette variable. On peut, en effet considérer le temps d'arrivée de la première goutte d'eau, de la vitesse maximale (souvent très proche), du débit maximal ou de la cote d'eau maximale. En rupture instantanée et à proximité du barrage, ces 4 temps sont très proches mais il n'en est pas de même en rupture progressive où, en outre, l'origine des temps est également dis- 


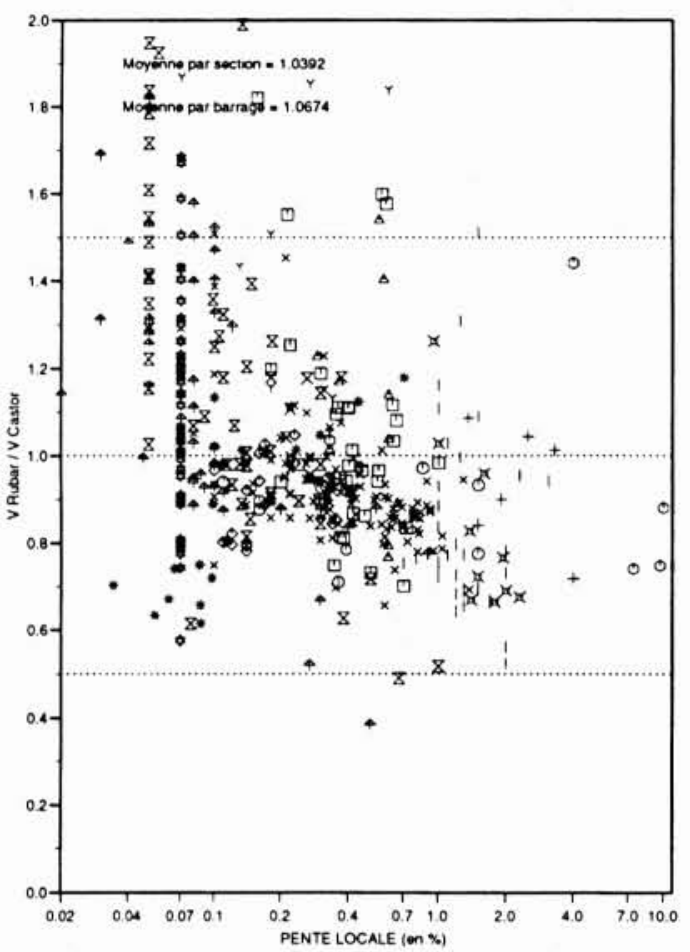

4. Vitesses maximales.

cutable (début de la rupture, instant où le débit de pointe est atteint au droit du barrage, ...).

CASTOR calcule un temps unique en 2 étapes :

- temps de formation de la brèche (exprimé en secondes) : nul en rupture instantanée, égal à 100 fois la hauteur du barrage (exprimée en mètres) en rupture progressive (d'après Wetmore et Fread [5]) ;

- temps de propagation qui doit être ajouté au temps précédent. Ce temps est obtenu à partir de l'estimation de la vitesse d'avancée du front comme moyenne des vitesses maximales au barrage et à la section considérée. La figure 5 montre l'ajustement obtenu sur ce temps de propagation. La dispersion est également très grande (écart-type : 0,34), une dizaine de sections ayant un rapport supérieur à 2 . Ce temps de propagation déterminé par CASTOR correspond en rupture instantanée au temps d'arrivée de la vitesse maximale et en rupture progressive à la durée entre l'instant du débit de pointe au barrage et l'instant du débit de pointe à la section considérée.

\section{UN EXEMPLE TRAITÉ PAR CASTOR}

Le cas choisi est celui du barrage de Lawn Lake aux Etats-Unis. Ce barrage correspond à un type relativement courant en France ( 8 mètres de haut pour une capacité de $830000 \mathrm{~m}^{3}$ ). Sa rupture par renard en juillet 1982 a entraîné la mort de 3 personnes et des dommages chiffrés à environ 200 millions de francs. Le débit au barrage a été estimé à $510 \mathrm{~m}^{3} / \mathrm{s}$. Quelques profils en travers étaient disponibles ainsi que des observations sur les cotes d'eau maximales atteintes et les temps d'obtention des cotes maximales [2].

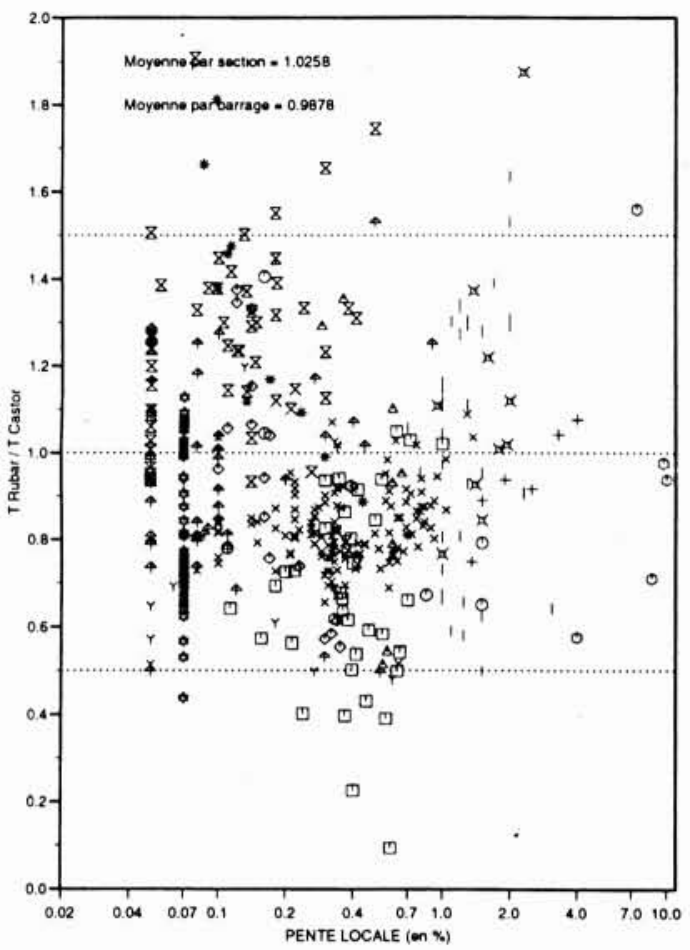

\section{Temps de propagation de l'onde.}

Les figures 6 et 7 effectuent la comparaison entre les observations, le calcul mené avec CASTOR (avec débit de pointe fixé à $510 \mathrm{~m}^{3} / \mathrm{s}$ ) et celui mené avec RUBAR 3

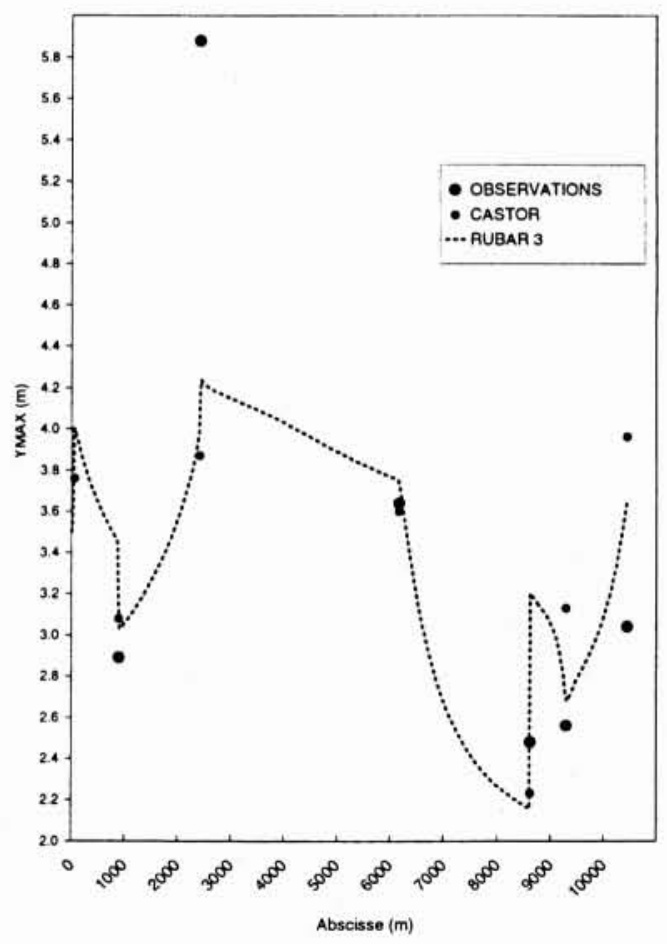

6. Hauteurs d'eau maximales (barrage de Lawn Lake). 


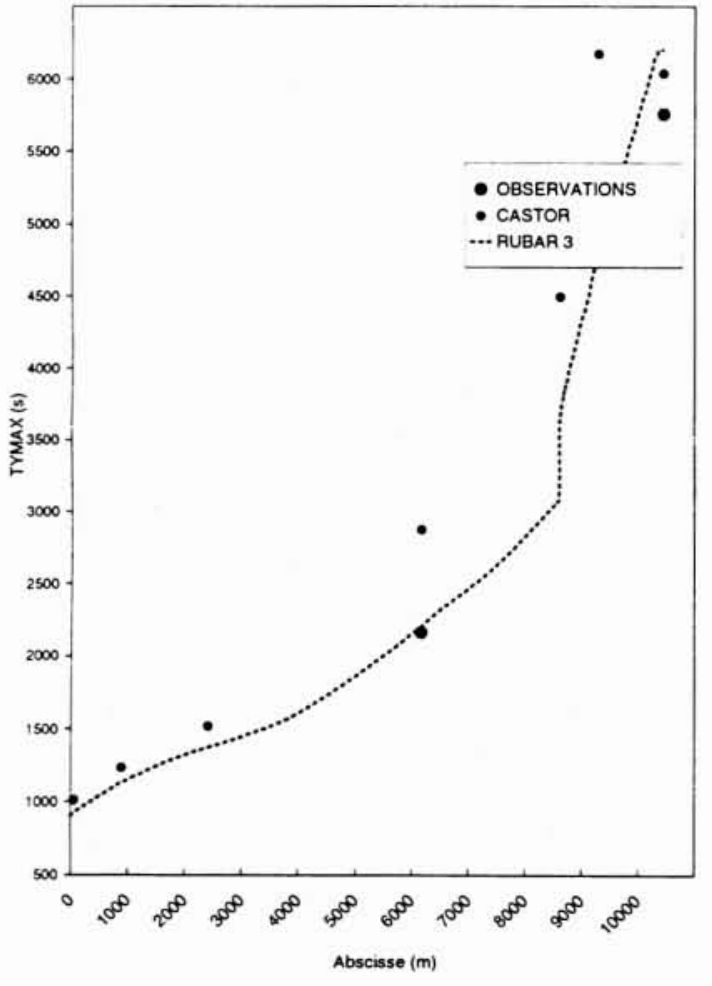

7. Temps des cotes maximales (barrage de Lawn Lake).

(résolution des équations de Saint-Venant à partir d'un hydrogramme au barrage obtenu par le logiciel RUPRO avec débit de pointe de $510 \mathrm{~m}^{3} / \mathrm{s}$ ).

On observe que :

- l'accord des 2 modèles avec les observations est globalement satisfaisant bien qu'on se situe aux limites d'utilisation (pente de l'ordre de $10 \%$, transport solide important) ;

- les 2 modèles sont très proches, les différences avec les observations se retrouvant aux mêmes endroits avec le même ordre de grandeur ;

- la hauteur d'eau maximale (ou plutôt la cote maximale) en l'abscisse 2400 est erronée. Les calculs effectués à partir de la topographie d'avant la rupture n'ont, en effet, pas pris en compte les importants dépôts de matériaux ayant eu lieu à cet endroit.

\section{CONCLUSIONS}

Le logiciel CASTOR se présente aujourd'hui comme un logiciel utilisable sur micro-ordinateur et accessible à des ingénieurs ou techniciens non spécialisés.
Dans le cadre de son domaine d'utilisation, la précision de la méthode peut être jugée comme satisfaisante: incertitude de l'ordre de $30 \%$ sur les hauteurs d'eau maximales et de $50 \%$ sur les autres valeurs obtenues (débit maximal, vitesse maximale et temps d'arrivée) si on retient l'intervalle où plus de $90 \%$ des résultats de notre échantillon se situent.

Ces résultats sont obtenus à partir d'un petit nombre de données; en particulier, la vallée n'est décrite que par sa pente moyenne et un coefficient de frottement (Strickler). Les calculs sont également très simples, la principale difficulté restant l'obtention du débit maximal, étape qui est résolue grâce à un abaque.

Le présent travail de recherche a, en effet, permis de montrer que l'équation du régime uniforme pouvait être utilisée localement pour passer du débit maximal à la hauteur d'eau maximale et à la vitesse maximale. En outre, le temps de propagation de l'onde est obtenu simplement en divisant la distance au barrage par la vitesse maximale moyenne sur le bief.

\section{Remerciements}

Nous remercions le Ministère Français de l'Environnement (Service de la Recherche, des Etudes et du Traitement de l'Information sur l'Environnement) pour le soutien financier apporté au travail de recherche accompli sur le logiciel CASTOR.

\section{Bibliographie}

[1] Colin E., Pochat R. (1978). - Calcul d'onde de submersion due à la rupture d'un barrage. CTGREF, $\mathrm{N}^{\circ} 25$, Etude.

[2] JARRetT R.D., CostA J.E. (1986), - Hydrology, Geomorphology and Dam-Break Modeling of the July 15, 1982 Lawn Lake Dam and Cascade Lake Dam Failures, Larimer County, Colorado. U.S. Geological Survey Profesional Paper 1369.

[3] Molinaro P., Maione D. (1991). - The experience developped by ENEL in dam-break wave computation and flood risk analysis. Colloque International pour la Simulation des Ondes de Submersion, Montréal.

[4] RoBIN O. (1990). - Méthode simplifiée de calcul d'onde de submersion résultant de la rupture d'un barrage. CEMAGREF, DEA de l'Université Claude Bernard.

[5] WETMORE J.N., FREAD D.L. (1983). - The NWS simplified dam break flood forecasting model for desk-top and handheld microcomputers. National Weather Service, Office of Hydrology.

[6] WURBS R.A. (1987). - Dam-breach flood wave models. Journal of Hydraulic Engineering, Vol. 113, $\mathrm{n}^{\circ} 1$. 